\title{
Impact of Price Display on Provider Ordering: A Systematic Review
}

\author{
Mark T. Silvestri, MD, MHS ${ }^{1 \star}$, Tasce R. Bongiovanni, MD, MHS ${ }^{1,2}$, Janis G. Glover, MLS³, Cary P. Gross, MD ${ }^{1,4}$
}

${ }^{1}$ Robert Wood Johnson Foundation Clinical Scholars Program, Yale School of Medicine, New Haven, Connecticut; ${ }^{2}$ U.S. Department of Veterans Affairs, Connecticut Healthcare System, West Haven, Connecticut; ${ }^{3}$ Harvey Cushing/John Hay Whitney Medical Library, Yale University, New Haven, Connecticut; ${ }^{4}$ Department of Internal Medicine, Yale School of Medicine, New Haven, Connecticut.

BACKGROUND: Displaying order prices to physicians is 1 potential strategy to reduce unnecessary health expenditures, but its impact on patterns of care is unclear.

OBJECTIVE: To review characteristics of previous price display interventions, impact on order costs and volume, effects on patient safety, acceptability to physicians, and the quality of this evidence.

DESIGN: Systematic review of studies that showed numeric prices of laboratory tests, imaging studies, or medications to providers in real time during the ordering process and evaluated the impact on provider ordering. Two investigators independently extracted data for each study and evaluated study quality using a modified Downs and Black checklist.

RESULTS: Of 1494 studies reviewed, 19 met inclusion criteria, including 5 randomized trials, 13 pre-post intervention studies, and 1 time series analysis. Studies were published between 1983 and 2014. Of 15 studies reporting the quantitative impact of price display on aggregate order costs or volume, 10 demonstrated a statistically significant decrease in the intervention group. Price display was found to decrease aggregate order costs (9 of 13 studies) more frequently than order volume (3 of 8 studies). Patient safety was evaluated in 5 studies and was unaffected by price display. Provider acceptability tended to be positive, although evidence was limited. Study quality was mixed, with checklist scores ranging from $5 / 21$ to $20 / 21$.

CONCLUSIONS: Provider price display likely reduces order costs to a modest degree. Patient safety appeared unchanged, though evidence was limited. More high-quality evidence is needed to confirm these findings within a modern context. Journal of Hospital Medicine 2016;11:65-76. (C) 2015 Society of Hospital Medicine
Rising healthcare spending has garnered significant public attention, and is considered a threat to other national priorities. Up to one-third of national health expenditures are wasteful, the largest fraction generated through unnecessary services that could be substituted for less-costly alternatives or omitted altogether. ${ }^{1}$ Physicians play a central role in health spending, as they "purchase" nearly all tests and therapies on behalf of patients.

One strategy to enhance cost-conscious physician ordering is to increase transparency of cost data for providers. $^{2-4}$ Although physicians consider price an important factor in ordering decisions, they have difficulty estimating costs accurately or finding price information easily. ${ }^{5,6}$ Improving physicians' knowledge of order costs may prompt them to forego diagnostic tests or therapies of low utility, or shift ordering to lower-cost alternatives. Real-time price display during provider order entry is 1 approach for achieving this goal. Modern electronic health records (EHRs) with

\footnotetext{
*Address for correspondence and reprint requests: Mark T. Silvestri, MD, Robert Wood Johnson Foundation Clinical Scholars Program, PO Box 208088, 333 Cedar Street, SHM IE-61, New Haven, CT 06520; Telephone: 617-947-9170; Fax: 203-785-3461; E-mail: mark.silvestri@yale.edu

Additional Supporting Information may be found in the online version of this article.

Received: June 2, 2015; Revised: August 18, 2015; Accepted: August 26, 2015

2015 Society of Hospital Medicine DOI 10.1002/jhm.2500

Published online in Wiley Online Library (Wileyonlinelibrary.com).
}

computerized physician order entry (CPOE) make price display not only practical but also scalable. Integrating price display into clinical workflow, however, can be challenging, and there remains lack of clarity about potential risks and benefits. The dissemination of real-time CPOE price display, therefore, requires an understanding of its impact on clinical care.

Over the past 3 decades, several studies in the medical literature have evaluated the effect of price display on physician ordering behavior. To date, however, there has been only 1 narrative review of this literature, which did not include several recent studies on the topic or formally address study quality and physician acceptance of price display modules. ${ }^{7}$ Therefore, to help inform healthcare leaders, technology innovators, and policy makers, we conducted a systematic review to address 4 key questions: (1) What are the characteristics of interventions that have displayed order prices to physicians in the context of actual practice? (2) To what degree does real-time display of order prices impact order costs and order volume? (3) Does price display impact patient safety outcomes, and is it acceptable to providers? (4) What is the quality of the current literature on this topic?

\section{METHODS \\ Data Sources}

We searched 2 electronic databases, MEDLINE and Embase, using a combination of controlled vocabulary terms and keywords that covered both the targeted intervention (eg, fees and charges) and the outcome of 
interest (eg, physician's practice patterns), limited to English language articles with no restriction on country or year of publication (see Supporting Information, Appendix 1, in the online version of this article). The search was run through August 2014. Results from both database searches were combined and duplicates eliminated. We also ran a MEDLINE keyword search on titles and abstracts of articles from 2014 that were not yet indexed. A medical librarian was involved in all aspects of the search process. ${ }^{8}$

\section{Study Selection}

Studies were included if they evaluated the effect of displaying actual order prices to providers during the ordering process and reported the impact on provider ordering practices. Reports in any clinical context and with any study design were included. To assess most accurately the effect of price display on real-life ordering and patient outcomes, studies were excluded if: (1) they were review articles, commentaries, or editorials; (2) they did not show order prices to providers; (3) the context was a simulation; (4) the prices displayed were relative (eg, $\$ / \$ \$ \$ \$ \$$ ) or were only cumulative; (5) prices were not presented real-time during the ordering process; or (6) the primary outcome was neither order costs nor order volume. We decided a priori to exclude simulations because these may not accurately reflect provider behavior when treating real patients, and to exclude studies showing relative prices due to concerns that it is a less significant price transparency intervention and that providers may interpret relative prices differently from actual prices.

Two reviewers, both physicians and health service researchers (M.T.S. and T.R.B.), separately reviewed the full list of titles and abstracts. For studies that potentially met inclusion criteria, full articles were obtained and were independently read for inclusion in the final review. The references of all included studies were searched manually, and the Scopus database was used to search all studies that cited the included studies. We also searched the references of relevant literature reviews. ${ }^{9-11}$ Articles of interest discovered through manual search were then subjected to the same process.

\section{Data Extraction and Quality Assessment}

Two reviewers (M.T.S. and T.R.B.) independently performed data extraction using a standardized spreadsheet. Discrepancies were resolved by reviewer consensus. Extracted study characteristics included study design and duration, clinical setting, study size, type of orders involved, characteristics of price display intervention and control, and type of outcome. Findings regarding patient safety and provider acceptability were also extracted when available.

Study quality was independently evaluated and scored by both reviewers using the Downs and Black checklist, designed to assess quality of both randomized and nonrandomized studies. ${ }^{12}$ The checklist contains 5 items pertaining to allocation concealment, blinding, or follow-up that are not applicable to an administrative intervention like price display, so these questions were excluded. Additionally, few studies calculated sample size or reported post hoc statistical power, so we also excluded this question, leaving a modified 21-item checklist. We also assessed each study for sources of bias that were not already assessed by the Downs and Black checklist, including contamination between study groups, confounding of results, and incomplete intervention or data collection.

\section{Data Synthesis}

Data are reported in tabular form for all included studies. Due to heterogeneity of study designs and outcome measures, data from the studies were not pooled quantitatively. This review is reported according to the Preferred Reporting Items for Systematic Reviews and Meta-Analysis guidelines.

\section{RESULTS}

Database searches yielded a total of 1400 articles, of which 18 were selected on the basis of title and abstract for detailed assessment. Reference searching led us to retrieve 94 further studies of possible interest, of which 23 were selected on the basis of abstract for detailed assessment. Thus, 41 publications underwent full manuscript review, 19 of which met all inclusion criteria (see Supporting Information, Appendix 2, in the online version of this article). ${ }^{13-31}$ These studies were published between 1983 and 2014, and were conducted primarily in the United States.

\section{Study Characteristics}

There was considerable heterogeneity among the 19 studies with regard to design, setting, and scope (Table $1)$. There were 5 randomized trials, for which the units of randomization were patient (1), provider team (2), and test (2). There were 13 pre-post intervention studies, 5 of which used a concomitant control group, and 2 of which included a washout period. There was 1 interrupted time series study. Studies were conducted within inpatient hospital floors (8), outpatient clinics (4), emergency departments (ED) or urgent care facilities (4), and hospital operating rooms (3).

Prices were displayed for laboratory tests (12 studies), imaging tests (8 studies), and medications (7 studies). Study scope ranged from examining a single medication class to evaluating all inpatient orders. The type of price used for the display varied, with the most common being the facility charges or "chargemaster prices" (6 studies), and Medicare prices (3 studies). In several cases, price display was only 1 component of the study, and 6 studies introduced additional interventions concurrent with price display, such as cost-effective ordering menus, ${ }^{29}$ medication 


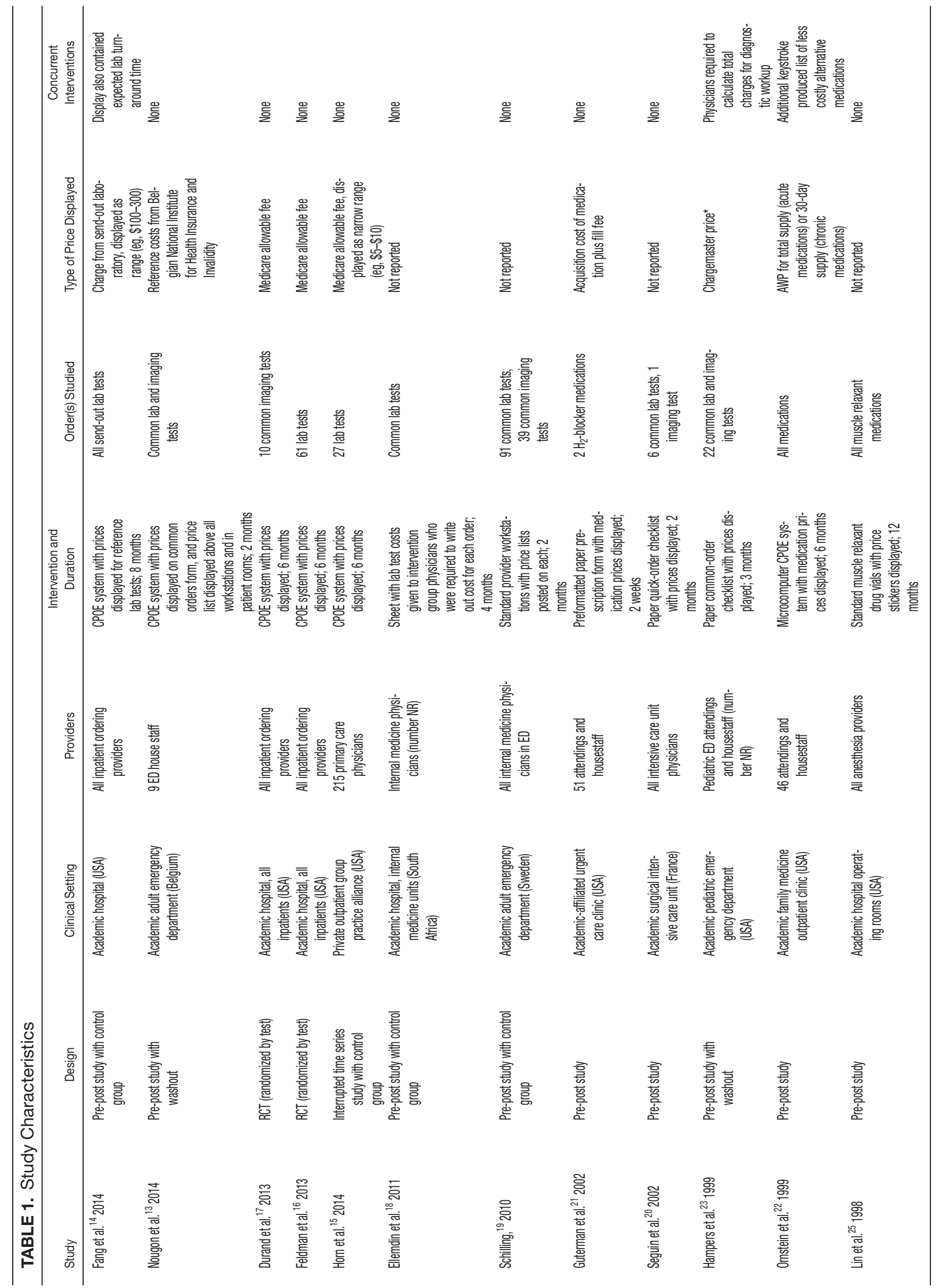




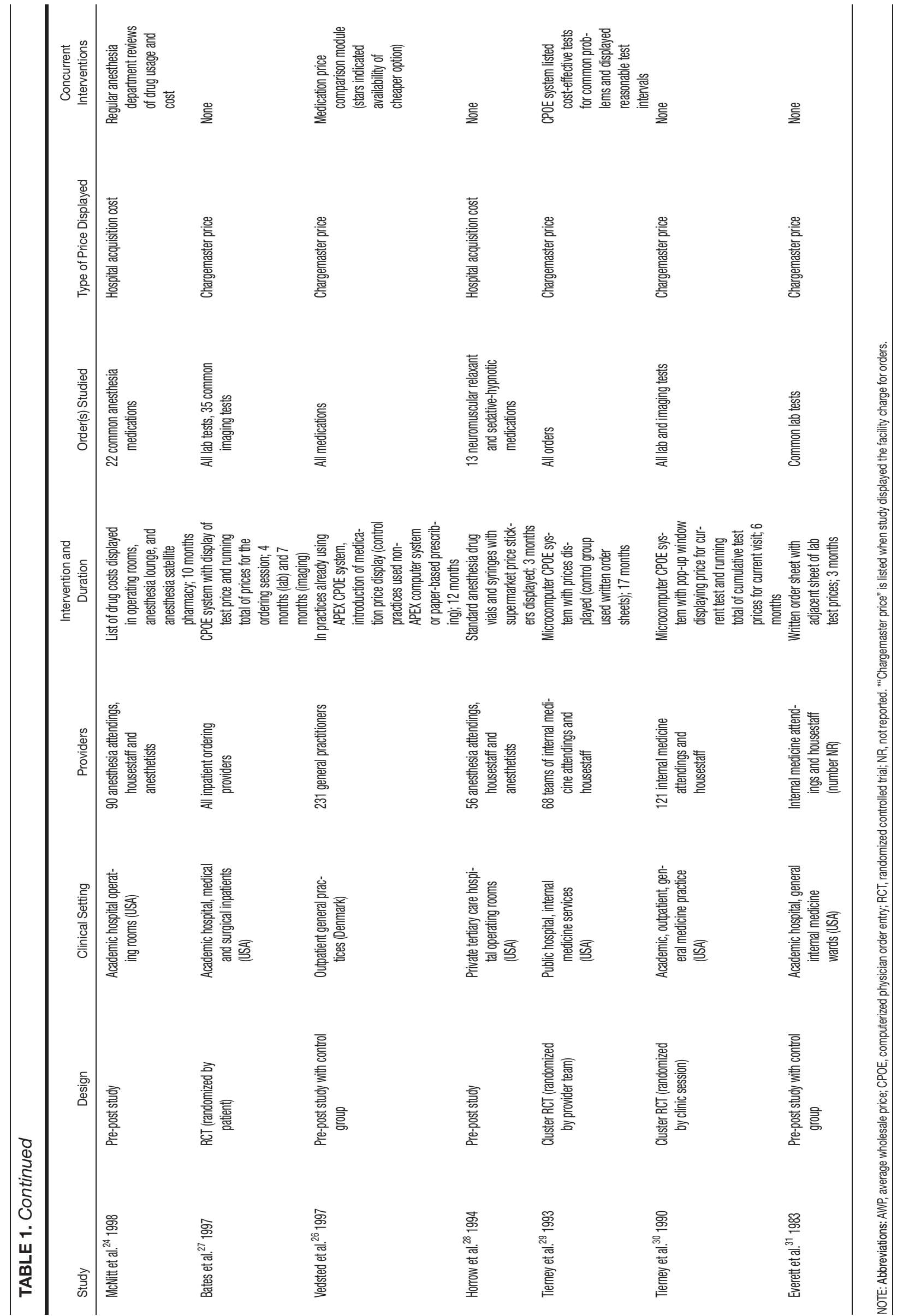




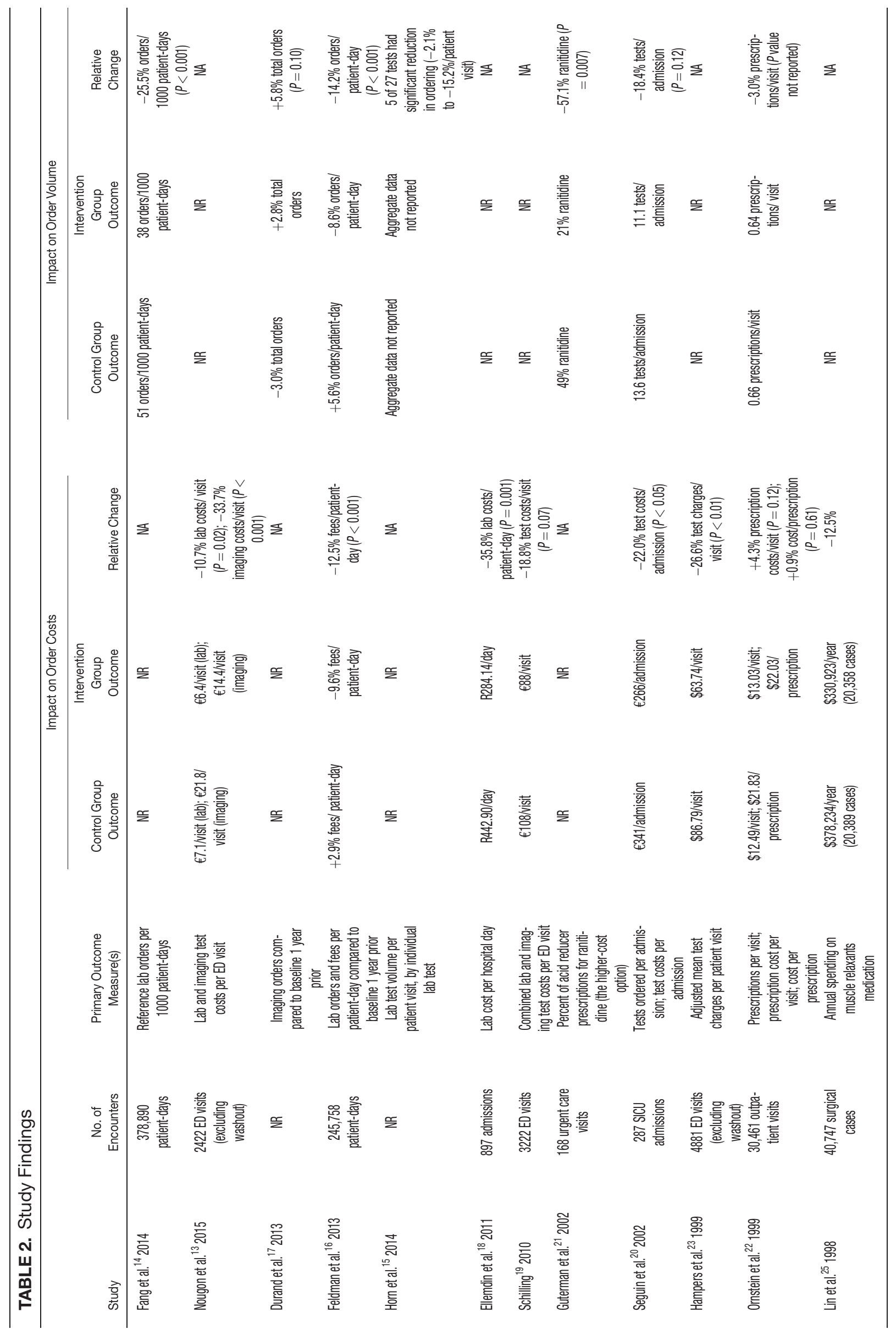




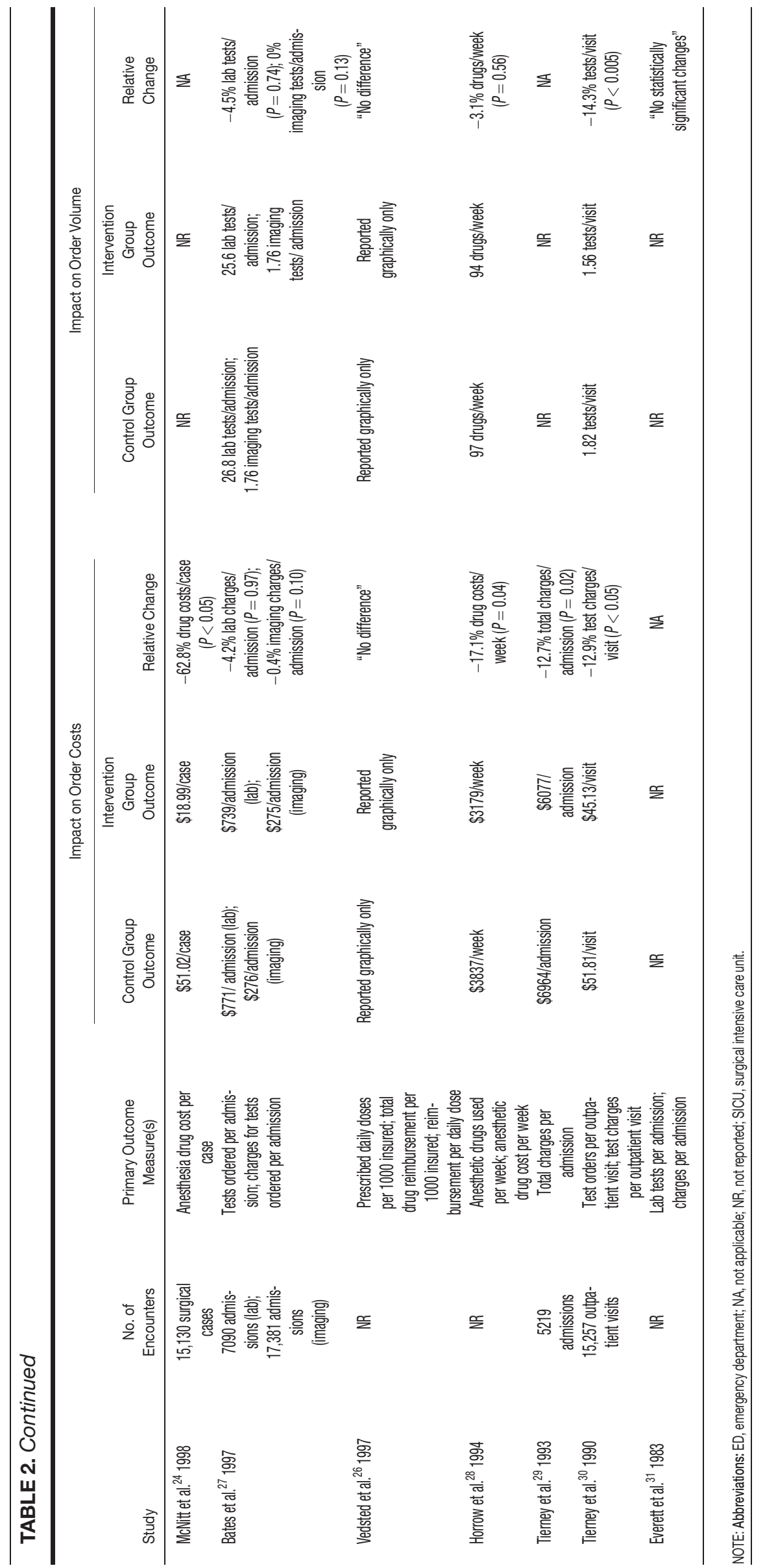




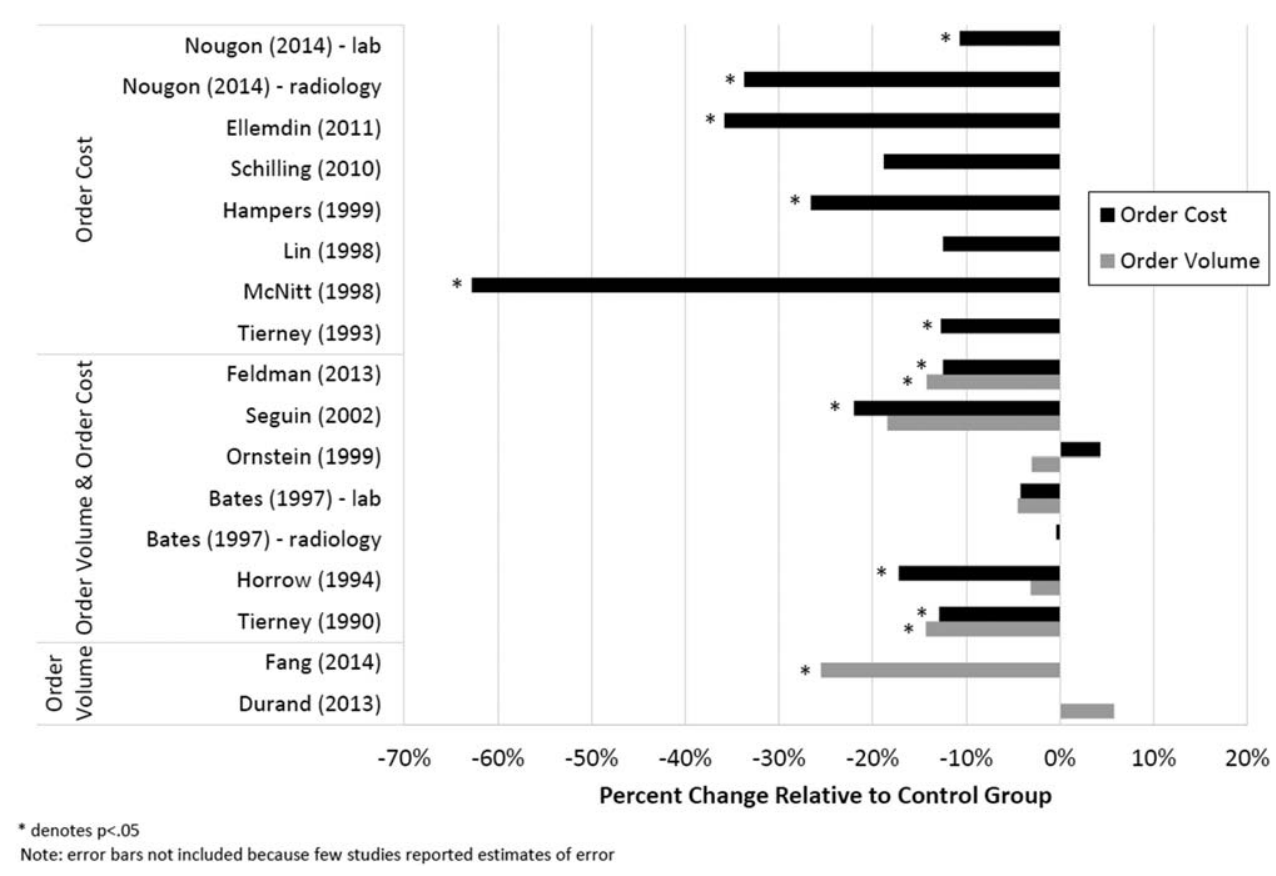

FIG. 1. Impact of price display on aggregate order costs and volume.

comparison modules, ${ }^{26}$ or display of test turnaround times. ${ }^{14}$ Seven of the 19 studies were conducted in the past decade, of which 5 displayed prices within an EHR. ${ }^{13-17}$

\section{Order Costs and Volume}

Thirteen studies reported the numeric impact of price display on aggregate order costs (Table 2). Nine of these demonstrated a statistically significant $(P<$ $0.05)$ decrease in order costs, with effect sizes ranging from $10.7 \%$ to $62.8 \%$. $^{13,16,18,20,23,24,28-30}$ Decreases were found for lab costs, imaging costs, and medication costs, and were observed in both the inpatient and outpatient settings. Three of these 9 studies were randomized. For example, in 1 study randomizing 61 lab tests to price display or no price display, costs for the intervention labs dropped $9.6 \%$ compared to the year prior, whereas costs for control labs increased $2.9 \%(P<0.001){ }^{16}$ Two studies randomized by provider group showed that providers seeing order prices accrued $12.7 \%$ fewer charges per inpatient admission $(P=0.02)$ and $12.9 \%$ fewer test charges per outpatient visit $(P<0.05) .{ }^{29,30}$ Three studies found no significant association between price display and order costs, with effect sizes ranging from a decrease of $18.8 \%$ to an increase of $4.3 \% .^{19,22,27}$ These studies also evaluated lab, imaging, and medication costs, and included 1 randomized trial. One additional large study noted a $12.5 \%$ decrease in medication costs after initiation of price display, but did not statistically evaluate this difference. ${ }^{25}$

Eight studies reported the numeric impact of price display on aggregate order volume. Three of these demonstrated a statistically significant decrease in order volume, with effect sizes ranging from $14.2 \%$ to $25.5 \%{ }^{14,16,30}$ Decreases were found for lab and imaging tests, and were observed in both inpatient and outpatient settings. For example, 1 pre-post study displaying prices for inpatient send-out lab tests demonstrated a $25.5 \%$ reduction in send-out labs per 1000 patient-days $(P<0.001)$, whereas there was no change for the control group in-house lab tests, for which prices were not shown. ${ }^{14}$ The other 5 studies reported no significant association between price display and order volume, with effect sizes ranging from a decrease of $18.4 \%$ to an increase of $5.8 \% .^{17,20,22,27,28}$ These studies evaluated lab, imaging, and medication volume. One trial randomizing by individual inpatient showed a nonsignificant decrease of $4.5 \%$ in lab orders per admission in the intervention group $(P=0.74)$, although the authors noted that their study had insufficient power to detect differences less than $10 \% .{ }^{27}$ Of note, 2 of the 5 studies reporting nonsignificant impacts on order volume $(-3.1 \%, P=0.56$; and $-18.4 \%, P=0.12)$ did demonstrate significant decreases in order costs $(-17.1 \%$, $P=0.04$; and $-22.0 \%, P<0.05) . .^{20,28}$

There were an additional 2 studies that reported the impact of price display on order volume for individual orders only. In 1 time-series study showing lab test prices, there was a statistically significant decrease in order volume for 5 of 27 individual tests studied (using a Bonferroni-adjusted threshold of significance), with no tests showing a significant increase. ${ }^{15}$ In 1 pre-post study showing prices for $\mathrm{H} 2$-antagonist drugs, there was a statistically significant $57.1 \%$ decrease in order volume for the high-cost medication, with a corresponding $58.7 \%$ increase in the low-cost 


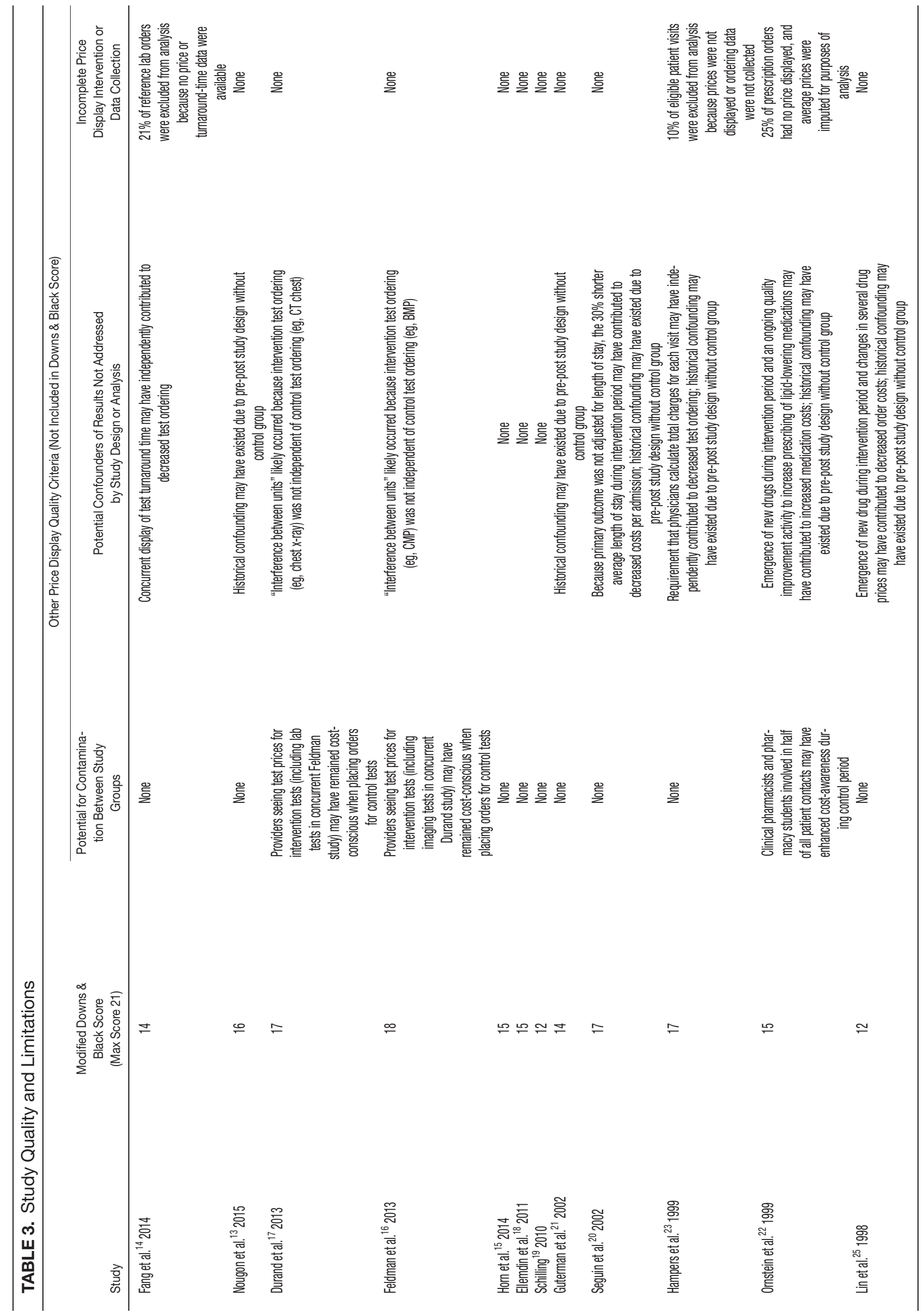




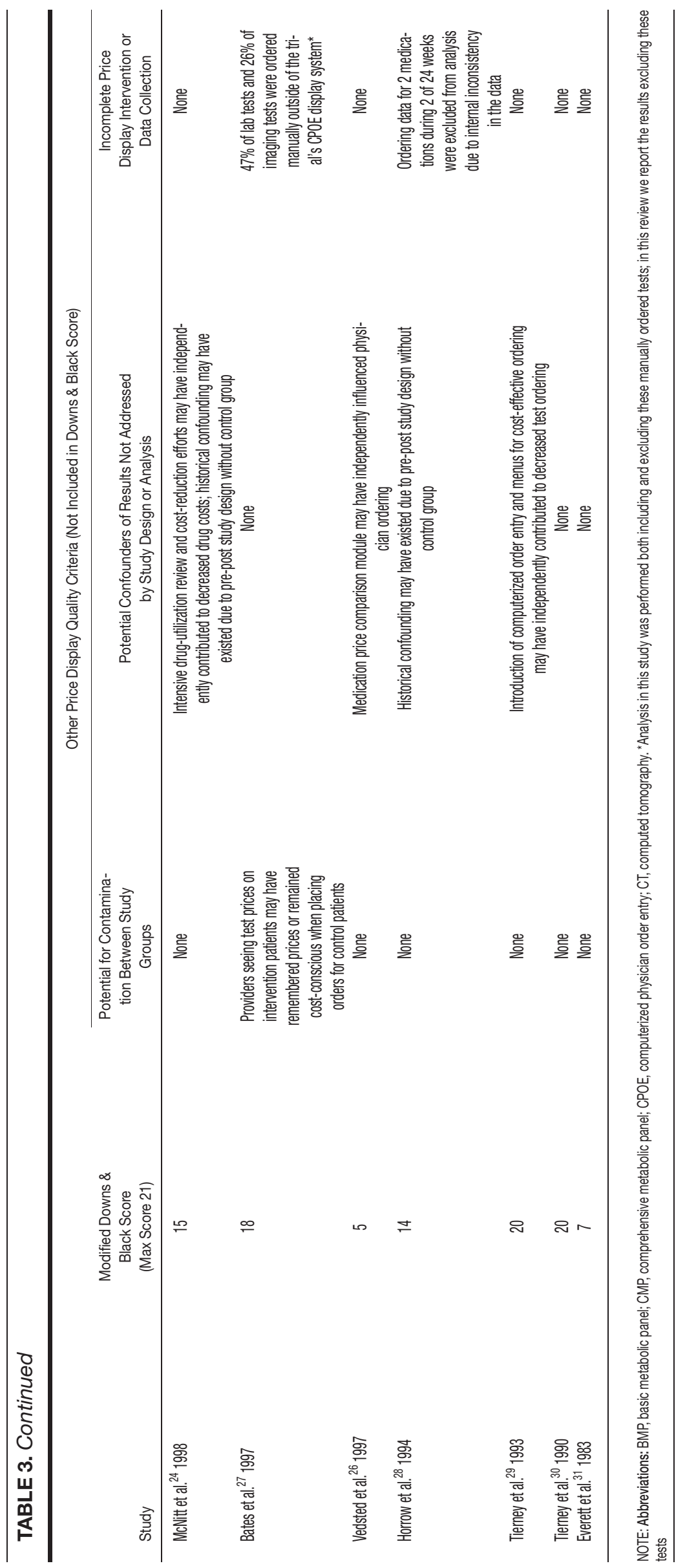


option. $^{21}$ These studies did not report impact on aggregate order costs. Two further studies in this review did not report outcomes numerically, but did state in their articles that significant impacts on order volume were not observed. ${ }^{26,31}$

Therefore, of the 19 studies included in this review, 17 reported numeric results. Of these 17 studies, 12 showed that price display was associated with statistically significant decreases in either order costs or volume, either in aggregate (10 studies; Figure 1) or for individual orders ( 2 studies). Of the 7 studies conducted within the past decade, 5 noted significant decreases in order costs or volume. Prices were embedded into an EHR in 5 of these recent studies, and 4 of the 5 observed significant decreases in order costs or volume. Only 2 studies from the past decade- 1 from Belgium and 1 from the United Statesincorporated prices into an EHR and reported aggregate order costs. Both found statistically significant decreases in order costs with price display. ${ }^{13,16}$

\section{Patient Safety and Provider Acceptability}

Five studies reported patient-safety outcomes. One inpatient randomized trial showed similar rates of postdischarge utilization and charges between the intervention and control groups. ${ }^{29}$ An outpatient randomized trial showed similar rates of hospital admissions, ED visits, and outpatient visits between the intervention and control groups. ${ }^{30}$ Two pre-post studies showing anesthesia prices in hospital operating rooms included a quality assurance review and showed no changes in adverse outcomes such as prolonged postoperative intubation, recovery room stay, or unplanned intensive care unit admissions. ${ }^{24,25}$ The only adverse safety finding was in a pre-post study in a pediatric ED, which showed a higher rate of unscheduled follow-up care during the intervention period compared to the control period $(24.4 \%$ vs $17.8 \%, P<0.01$ ) but similar rates of patients "feeling better" $(83.4 \%$ vs $86.7 \%, P=0.05)$. These findings, however, were based on self-report during telephone follow-up with a $47 \%$ response rate. ${ }^{23}$

Five studies reported on provider acceptability of price display. Two conducted questionnaires as part of the study plan, whereas the other 3 offered general provider feedback. One questionnaire revealed that $83 \%$ of practices were "satisfied" or "very satisfied" with the price display. ${ }^{26}$ The other questionnaire found that $81 \%$ of physicians felt the price display "improved my knowledge of the relative costs of tests I order" and similarly $81 \%$ "would like additional cost information displayed" for other orders. ${ }^{15}$ Three studies reported subjectively that showing prices "initially caused questions from most physicians," 13 but that ultimately, physicians "like seeing this information" 27 and gave feedback that was "generally positive." 21 One study evaluated the impact of price display on provider cost knowledge. Providers in the intervention group did not improve in their costawareness, with average errors in cost estimates exceeding $40 \%$ even after 6 months of price display. ${ }^{30}$

\section{Study Quality}

Using a modified Downs and Black checklist of 21 items, studies in this review ranged in scores from 5 to 20 , with a median score of 15 . Studies most frequently lost points for being nonrandomized, failing to describe or adjust for potential confounders, being prone to historical confounding, or not evaluating potential adverse events.

We supplemented this modified Downs and Black checklist by reviewing 3 categories of study limitations not well-reflected in the checklist scoring (Table 3). The first was potential for contamination between study groups, which was a concern in 4 studies. For example, 1 pre-post study assessing medication ordering included clinical pharmacists in patient encounters both before and after the price display intervention. ${ }^{22}$ This may have enhanced cost-awareness even before prices were shown. The second set of limitations, present in 12 studies, included confounders that were not addressed by study design or analysis. For example, the intervention in 1 study displayed not just test cost but also test turnaround time, which may have separately influenced providers against ordering a particular test. ${ }^{14}$ The third set of limitations included unanticipated gaps in the display of prices or in the collection of ordering data, which occurred in 5 studies. If studies did not report on gaps in the intervention or data collection, we assumed there were none.

Even among the 5 randomized trials there were substantial limitations. For example, 2 trials used individual tests as the unit of randomization, although ordering patterns for these tests are not independent of each other (eg, ordering rates for comprehensive metabolic panels are not independent of ordering rates for basic metabolic panels). ${ }^{16,17}$ This creates "interference between units" that was not accounted for in the analysis. ${ }^{32}$ A third trial was randomized at the level of the patient, so was subject to contamination as providers seeing the price display for intervention group patients may have remained cost-conscious while placing orders for control group patients. ${ }^{27}$ In a fourth trial, the measured impact of the price display may have been confounded by other aspects of the overall cost intervention, which included cost-effective test menus and suggestions for reasonable testing intervals. ${ }^{29}$

The highest-quality study was a cluster-randomized trial published in 1990 specifically measuring the effect of price display on a wide range of orders. ${ }^{30}$ Providers and patients were separated by clinic session so as to avoid contamination between groups, and the trial included more than 15,000 outpatient visits. The intervention group providers ordered $14.3 \%$ fewer 
tests than control group providers, which resulted in $12.9 \%$ lower charges.

\section{DISCUSSION}

We identified 19 published reports of interventions that displayed real-time order prices to providers and evaluated the impact on provider ordering. There was substantial heterogeneity in study setting, design, and quality. Although there is insufficient evidence on which to base strong conclusions, these studies collectively suggest that provider price display likely reduces order costs to a modest degree. Data on patient safety were largely lacking, although in the few studies that examined patient outcomes, there was little evidence that patient safety was adversely affected by the intervention. Providers widely viewed display of prices positively.

Our findings align with those of a recent systematic review that concluded that real-time price information changed provider ordering in the majority of studies. ${ }^{7}$ Whereas that review evaluated 17 studies from both clinical settings and simulations, our review focused exclusively on studies conducted in actual ordering environments. Additionally, our literature search yielded 8 studies not previously reviewed. We believe that the alignment of our findings with the prior review, despite the differences in studies included, adds validity to the conclusion that price display likely has a modest impact on reducing order costs. Our review contains several additions important for those considering price display interventions. We provide detailed information on study settings and intervention characteristics. We present a formal assessment of study quality to evaluate the strength of individual study findings and to guide future research in this area. Finally, because both patient safety and provider acceptability may be a concern when prices are shown, we describe all safety outcomes and provider feedback that these studies reported.

The largest effect sizes were noted in 5 studies reporting decreases in order volume or costs greater than $25 \% .^{13,14,18,23,24}$ These were all pre-post intervention studies, so the effect sizes may have been exaggerated by historical confounding. However, the 2 studies with concurrent control groups found no decreases in order volume or cost in the control group. ${ }^{14,18}$ Among the 5 studies that did not find a significant association between price display and provider ordering, 3 were subject to contamination between study groups, ${ }^{17,22,27}$ 1 was underpowered, ${ }^{19}$ and 1 noted a substantial effect size but did not perform a statistical analysis. ${ }^{25} \mathrm{We}$ also found that order costs were more frequently reduced than order volume, likely because shifts in ordering to less expensive alternatives may cause costs to decrease while volume remains unchanged. ${ }^{20,28}$

If price display reduces order costs, as the majority of studies in this review indicate, this finding carries broad implications. Policy makers could promote cost- conscious care by creating incentives for widespread adoption of price display. Hospital and health system leaders could improve transparency and reduce expenses by prioritizing price display. The specific beneficiaries of any reduced spending would depend on payment structures. With shifts toward financial riskbearing arrangements like accountable care organizations, healthcare institutions may have a financial interest in adopting price display. Because price display is an administrative intervention that can be developed within EHRs, it is potentially 1 of the most rapidly scalable strategies for reducing healthcare spending. Even modest reductions in spending on laboratory tests, imaging studies, and medications would result in substantial savings on a system-wide basis.

Implementing price display does not come without challenges. Prices need to be calculated or obtained, loaded into an EHR system, and updated periodically. Technology innovators could enhance EHR software by making these processes easier. Healthcare institutions may find displaying relative prices (eg, $\$ / \$ \$ \$ \$ \$$ ) logistically simpler in some contexts than showing actual prices (eg, purchase cost), such as when contracts require prices to be confidential. Although we decided to exclude studies displaying relative prices, our search identified no studies that met other inclusion criteria but displayed relative prices, suggesting a lack of evidence regarding the impact of relative price display as an alternative to actual price display.

There are 4 key limitations to our review. First, the heterogeneity of the study designs and reported outcomes precluded pooling of data. The variety of clinical settings and mechanisms through which prices were displayed enhances the generalizability of our findings, but makes it difficult to identify particular contexts (eg, type of price or type of order) in which the intervention may be most effective. Second, although the presence of negative studies on this subject reduces the concern for reporting bias, it remains possible that sites willing to implement and study price displays may be inherently more sensitive to prices, such that published results might be more pronounced than if the intervention were widely implemented across multiple sites. Third, the mixed study quality limits the strength of conclusions that can be drawn. Several studies with both positive and negative findings had issues of bias, contamination, or confounding that make it difficult to be confident of the direction or magnitude of the main findings. Studies evaluating price display are challenging to conduct without these limitations, and that was apparent in our review. Finally, because over half of the studies were conducted over 15 years ago, it may limit their generalizability to modern ordering environments.

We believe there remains a need for high-quality evidence on this subject within a contemporary context to confirm these findings. The optimal methodology for evaluating this intervention is a cluster 
randomized trial by facility or provider group, similar to that reported by Tierney et al. in 1990, with a primary outcome of aggregate order costs. ${ }^{30}$ Given the substantial investment this would require, a large time series study could also be informative. As most prior price display interventions have been under 6 months in duration, it would be useful to know if the impact on order costs is sustained over a longer time period. The concurrent introduction of any EHR alerts that could impact ordering (eg, duplicate test warnings) should be simultaneously measured and reported. Studies also need to determine the impact of price display alone compared to "price comparison" displays (displaying prices for the selected order along with reasonable alternatives). Although price comparison was a component of the intervention in some of the studies in this review, it was not evaluated relative to price display alone. Furthermore, it would be helpful to know if the type of price displayed affects its impact. For instance, if providers are most sensitive to the absolute magnitude of prices, then displaying chargemaster prices may impact ordering more than showing hospital costs. If, however, relative prices are all that providers need, then showing lower numbers, such as Medicare prices or hospital costs, may be sufficient. Finally, it would be reassuring to have additional evidence that price display does not adversely impact patient outcomes.

Although some details need elucidation, the studies synthesized in this review provide valuable data in the current climate of increased emphasis on price transparency. Although substantial attention has been devoted by the academic community, technology start-ups, private insurers, and even state legislatures to improving price transparency to patients, less focus has been given to physicians, for whom healthcare prices are often just as opaque. ${ }^{4}$ The findings from this review suggest that provider price display may be an effective, safe, and acceptable approach to empower physicians to control healthcare spending.

Disclosures: Dr. Silvestri, Dr. Bongiovanni, and Ms. Glover have nothing to disclose. Dr. Gross reports grants from Johnson \& Johnson, Medtronic Inc., and 21st Century Oncology during the conduct of this study. In addition, he received payment from Fair Health Inc. and ASTRO outside the submitted work.

\section{References}

1. Institute of Medicine. Best Care at Lower Cost: The Path to Continuously Learning Health Care in America: Washington, DC: National Academies Press; 2012.

2. Brook RH. Do physicians need a "shopping cart" for health care services? JAMA. 2012;307(8):791-792.

3. Reinhardt UE. The disruptive innovation of price transparency in health care. JAMA. 2013;310(18):1927-1928.

4. Riggs KR, DeCamp M. Providing price displays for physicians: which price is right? JAMA. 2014;312(16):1631-1632.

5. Allan GM, Lexchin J. Physician awareness of diagnostic and nondrug therapeutic costs: a systematic review. Int J Tech Assess Health Care. 2008;24(2):158-165.
6. Allan GM, Lexchin J, Wiebe N. Physician awareness of drug cost: a systematic review. PLoS Med. 2007;4(9):e283.

7. Goetz C, Rotman SR, Hartoularos G, Bishop TF. The effect of charge display on cost of care and physician practice behaviors: a systematic review. J Gen Intern Med. 2015;30:835-842.

8. Rethlefsen ML, Murad MH, Livingston EH. Engaging medical librarians to improve the quality of review articles. JAMA. 2014;312(10): 999-1000.

9. Axt-Adam P, van der Wouden JC, van der Does E. Influencing behavior of physicians ordering laboratory tests: a literature study. Med Care. 1993;31(9):784-794.

10. Beilby JJ, Silagy CA. Trials of providing costing information to general practitioners: a systematic review. Med J Aust. 1997;167(2):8992.

11. Grossman RM. A review of physician cost-containment strategies for laboratory testing. Med Care. 1983;21(8):783-802.

12. Downs SH, Black N. The feasibility of creating a checklist for the assessment of the methodological quality both of randomised and non-randomised studies of health care interventions. J Epidemiol Community Health. 1998;52(6):377-384.

13. Nougon G, Muschart X, Gerard V, et al. Does offering pricing information to resident physicians in the emergency department potentially reduce laboratory and radiology costs? Eur J Emerg Med. 2015;22: 247-252.

14. Fang DZ, Sran G, Gessner D, et al. Cost and turn-around time display decreases inpatient ordering of reference laboratory tests: a time series. BMJ Qual Saf. 2014;23:994-1000.

15. Horn DM, Koplan KE, Senese MD, Orav EJ, Sequist TD. The impact of cost displays on primary care physician laboratory test ordering. J Gen Intern Med. 2014;29:708-714.

16. Feldman LS, Shihab HM, Thiemann D, et al. Impact of providing fee data on laboratory test ordering: a controlled clinical trial. JAMA Intern Med. 2013;173(10):903-908.

17. Durand DJ, Feldman LS, Lewin JS, Brotman DJ. Provider cost transparency alone has no impact on inpatient imaging utilization. J Am Coll Radiol. 2013;10(2):108-113.

18. Ellemdin S, Rheeder P, Soma P. Providing clinicians with information on laboratory test costs leads to reduction in hospital expenditure. $S$ Afr Med J. 2011;101(10):746-748.

19. Schilling U. Cutting costs: the impact of price lists on the cost development at the emergency department. Eur J Emerg Med. 2010;17(6): 337-339.

20. Seguin P, Bleichner JP, Grolier J, Guillou YM, Malledant Y. Effects of price information on test ordering in an intensive care unit. Intens Care Med. 2002;28(3):332-335.

21. Guterman JJ, Chernof BA, Mares B, Gross-Schulman SG, Gan PG, Thomas D. Modifying provider behavior: a low-tech approach to pharmaceutical ordering. J Gen Intern Med. 2002;17(10):792-796.

22. Ornstein SM, MacFarlane LL, Jenkins RG, Pan Q, Wager KA. Medication cost information in a computer-based patient record system. Impact on prescribing in a family medicine clinical practice. Arch Fam Med. 1999;8(2):118-121.

23. Hampers LC, Cha S, Gutglass DJ, Krug SE, Binns HJ. The effect of price information on test-ordering behavior and patient outcomes in a pediatric emergency department. Pediatrics. $1999 ; 103(4$ pt 2):877882.

24. McNitt J, Bode E, Nelson R. Long-term pharmaceutical cost reduction using a data management system. Anesth Analg. 1998;87(4):837842.

25. Lin YC, Miller SR. The impact of price labeling of muscle relaxants on cost consciousness among anesthesiologists. J Clin Anesth. 1998; 10(5):401-403.

26. Vedsted P, Nielsen JN, Olesen F. Does a computerized price comparison module reduce prescribing costs in general practice? Fam Pract. 1997;14(3):199-203.

27. Bates DW, Kuperman GJ, Jha A, et al. Does the computerized display of charges affect inpatient ancillary test utilization? Arch Intern Med. 1997;157(21):2501-2508.

28. Horrow JC, Rosenberg H. Price stickers do not alter drug usage. Can I Anaesth. 1994:41(11):1047-1052.

29. Tierney WM, Miller ME, Overhage JM, McDonald CJ. Physician inpatient order writing on microcomputer workstations. Effects on resource utilization. JAMA. 1993;269(3):379-383.

30. Tierney WM, Miller ME, McDonald CJ. The effect on test ordering of informing physicians of the charges for outpatient diagnostic tests. $N$ Engl J Med. 1990;322(21):1499-1504.

31. Everett GD, deBlois CS, Chang PF, Holets T. Effect of cost education, cost audits, and faculty chart review on the use of laboratory services. Arch Intern Med. 1983;143(5):942-944.

32. Rosenbaum PR. Interference between units in randomized experiments. J Am Stat Assoc. 2007;102(477):191-200. 